\title{
ROSAT HRI OBSERVATIONS OF M33
}

\author{
Eric Schulman and Joel N. Bregman \\ Department of Astronomy, University of Michigan, Ann Arbor, MI 48109-1090 \\ eric@astro.lsa.umich.edu, jbregman@asrto.lsa.umich.edu
}

\begin{abstract}
Our $35 \mathrm{ksec}$ ROSAT HRI observation of M33 reveals $37 \mathrm{X}$-ray sources stronger than about $2.3 \sigma$. Eight of the sources are coincident with supernova remnants, four are coincident with giant HII regions, and three are coincident with HI holes. M33 X-7 is a compact accreting eclipsing binary, similar to binary X-ray sources detected in the Galaxy. Our ROSAT data confirm the binary interpretation and allow us to measure the period to an accuracy of $0.001 \%$. The nuclear source, $\mathrm{M} 33 \mathrm{X}-8$, is not found to be variable in the ROSAT HRI observations, although it varied as much as $40 \%$ between Einstein HRI observations.
\end{abstract}

\section{Observations and Data Reduction}

We observed M33 for $35 \mathrm{ksec}$ with the High-Resolution Imager (HRI) on ROSAT in $1992 \mathrm{Jan}$ and $1992 \mathrm{Aug}$. The two observation sections have pointing centers which differ by about $5^{\prime \prime}$ so the August observations were shifted before being merged with the January observations. Sources were found using ldetect as well as by determining the count rates at the coordinates of HI holes (Deul $\&$ den Hartog 1990) and supernova remnants (Long et al. 1990). We find $37 \mathrm{X}$-ray sources stronger than about $2.3 \sigma$, of which eight are coincident with supernova remnants, four are coincident with giant $H I I$ regions, and three are coincident with HI holes. Twelve of the sources were previously detected with Einstein Observatory observations. Two Einstein sources, M33 X-12 and M33 X-15 (according to the classification of Trinchieri, Fabbiano, \& Peres 1988 and Markert \& Rallis 1983), were not detected with ROSAT, despite the much higher sensitivity of the observations.

\section{Variability Analysis}

We studied variability within the ROSAT observations with three independent methods: the Cramer-Smirnov-Von Mises method and the KolmogorovSmirnov method (Eadie et al. 1971) which compare the cumulative distribution of photon arrival times with the distribution expected from a constant source, and a modified $\chi^{2}$ test able to provide binning independent results (Collura et al. 1987). All three tests indicate that M33 X-7 is variable at the $99.999 \%$ confidence level. We find no convincing evidence for variability in the other 14 sources that are strong enough to perform the variability analysis upon.

\section{An Eclipsing Binary X-Ray Source}

M33 X-7 was interpreted to be an eclipsing binary by Peres et al. (1989) based on Einstein observations. We confirm this interpretation and are able, because of the 12-year gap between the Einstein and ROSAT observations, to 
determine a much more accurate period, which we find to be $1.78572 \pm 0.00001$ days. The period we determine for M33 X-7 is very close to that of Her X-1 (Tananbaum et al. 1972) and the low phase lasts about a quarter of the period as in Cen X-3 (Schreier et al. 1972). A more thorough analysis of this source is presented in Schulman et al. (1993).

\section{The Nuclear Source}

The nuclear source, M33 X-8, does not appear to exhibit X-ray variability. We find the $3 \sigma$ amplitude upper limit for variability to be $6 \%$ on timescales of 8700 seconds, and $17 \%$ on timescales of 40 seconds. It is puzzling that the $\mathrm{X}$-ray flux changed by less than $1 \%$ between $1992 \mathrm{Jan}$ and $1992 \mathrm{Aug}$, since it decreased by $40 \%$ between $1979 \mathrm{Aug}$ and $1980 \mathrm{Jan}$, and increased by $20 \%$ between 1980 Jan and 1980 Aug (Peres et al. 1989).

The origin of the X-ray emission from M33 X-8 has been a mystery for some time (Markert \& Rallis 1983). Its X-ray luminosity of about $10^{39} \mathrm{erg} \mathrm{s}^{-1}$ is low for an AGN but quite high for a Galactic X-ray source, although a number of sources outside the Local Group have been found with comparable or larger $\mathrm{X}$-ray luminosities (most recently, Collura et al. (1994) determined the X-ray luminosity of a source in M82 to be at least $5.0 \times 10^{39} \mathrm{erg} \mathrm{s}^{-1}$ ). The nucleus has no detected $6 \mathrm{~cm}$ emission, no hydrogen line emission observed, little or no forbidden line emission, and infrared colors quite unlike those of AGN. Yet the young stars in the nucleus make up only a small fraction of the young population of M33, so that the a priori probability of a binary in the nucleus with an X-ray luminosity ten times that of any other source in M33 is small.

We thank Alfonso Collura, Fabio Reale, and Giovanni Peres for their help with the variability analysis. This research was supported by NASA through grant NAGW-2135 and through NASA Graduate Student Researchers Program Fellowship NGT-50901.

\section{References}

Collura, A., Maggio, A., Sciortino, S., Serio, S., Vaiana, G. S., \& Rosner, R. 1987, ApJ, 315, 340

Collura, A., Reale, F., Schulman, E., \& Bregman, J. N. 1994, ApJ (Letters), in press

Deul, E. R., \& den Hartog, R. H. 1990, A\&A, 229, 362

Eadie, W. T., Drijard, D., James, F., Roos, M., \& Sadoulet, B. 1971, Statistical Methods in Experimental Physics (Amsterdam: North-Holland)

Long, K. S., Blair, W. P., Kirshner, R. P, \& Winkler, P. F. 1990, ApJS, 72, 61.

Markert, T. H., \& Rallis, A. D. 1983, ApJ, 275, 571

Peres, G., Reale, F., Collura, A., \& Fabbiano, G. 1989, ApJ, 336, 140

Schreier, E., Levinson, R., Gursky, H., Kellogg, E. M., Tananbaum, H., \& Giacconi, R. 1972, ApJ, 172, L79

Schulman, E., Bregman, J. N., Collura, A., Reale, F., \& Peres, G. 1993, ApJ, 418, L67

Tananbaum, H., Gursky, H., Kellogg, E. M., Levinson, R., Schreier, E., \& Giacconi R. 1972, ApJ, 174, L143

Trinchieri, G., Fabbiano, G., \& Peres, G., 1988, ApJ, 325, 531 